RUNNING HEAD: SHAME TRAUMATIC MEMORIES IN EATING DISORDERS

Eating disorders: When social ranking is shaped by early shame experiences

\author{
Marcela Matos, $\mathrm{PhD} .^{* 1}$ \\ Cláudia Ferreira, PhD.* \\ Cristiana Duarte, MSc., PhD. Student* \\ José Pinto-Gouveia, MD., PhD. *
}

* Cognitive and Behavioural Research Centre, University of Coimbra

Correspondence concerning this manuscript should be addressed to:

Marcela Matos

CINEICC, Faculdade de Psicologia e Ciências da Educação, Universidade de Coimbra

Rua do Colégio Novo, Apartado 6153

3001-802 Coimbra, Portugal

Telefone: (+351) 239851450

Fax: $(+351) 239851462$

Email: marcela.s.matos@gmail.com

Acknowledgments: Part of this research has been supported by the first author (Marcela Matos) PostDoctoral grant number SFRH/BPD/84185/2012, sponsored by FCT (Portuguese Foundation for Science and Technology). 


\title{
Eating disorders: When social rank perceptions are shaped by early shame experiences
}

\begin{abstract}
Objectives: Recent research has shown that early adverse experiences can be an important risk factor for eating disorders. However, the role of early shame memories and how they are structured as traumatic and central memories to self-identity in eating disorders has never been investigated. The current study explores the phenomenology of shame memories involving attachment figures and other agents from the wider social domain in eating disorders patients, and examines the relationship between their traumatic and centrality features, social comparison based on physical appearance and the severity of eating psychopathology.
\end{abstract}

Design: The study has a cross-sectional design using a structured interview to assess retrospective data.

Methods: Participants in this study were 36 patients with an eating disorder diagnosis, who were assessed using Eating Disorder Examination (EDE 16.0D) and the Shame Experiences Interview (SEI) and self-report instruments measuring the traumatic features and centrality to identity of shame memories and social comparison.

Results: Results indicated that the traumatic and centrality qualities of shame memories involving social agents other than attachment figures (e.g., peers) are significantly correlated with unfavorable social rank perceptions and with increased eating psychopathology severity. Furthermore, the impact of such shame memories on eating psychopathology is fully mediated by social comparisons based on physical appearance. Conclusions: These findings highlight the importance of early shame experiences involving peers and other social agents that become traumatic memories, central to self- 
identity, to eating disorders patients' perceptions of inferiority and their core psychopathology features.

\section{Practitioner points}

- Early shame experiences of patients with eating disorders should be carefully assessed.

- Shame traumatic memories involving social agents outside the family circle (e.g., peers) seem to be especially damaging for patients with eating disorders, and influence how physical appearance is used as a central dimension to establish their social rank.

- Practitioners should directly work with these shame memories, focusing on diminishing their traumatic nature and reconstruct their central meaning to selfidentity.

Keywords: Eating disorders; Shame memories; Traumatic memory; Centrality to identity; Social comparison 


\section{Eating disorders: When social rank perceptions are shaped by early shame experiences}

\section{Introduction}

Eating disorders are an important public health problem, involving great levels of psychological and social impairment and posing significant health risks (Fairburn \& Harrison, 2003; Herzog, Deter, Fiehn, \& Petzold, 1997). The three main eating disorders diagnoses - anorexia nervosa, bulimia nervosa and eating disorders not otherwise specified - share a distinctive core psychopathology, which refers to the overevaluation of shape and weight and their control (Fairburn \& Harrison, 2003). This means that patients with eating disorders determine their self-worth largely, or even solely, based on their body, and judge themselves according to their ability to control their eating, weight and shape, intensively fearing losing control over these aspects (Fairburn, 2008).

It is widely accepted that there are multiple risk pathways for eating psychopathology. Recent research has emphasised the role that interpersonal sensitivities and perceptions of being in an unsafe social position in relation to one's group play in the development and maintenance of eating disorders (Goss \& Gilbert, 2002; Pinto-Gouveia, Ferreira, \& Duarte, 2012).

Physical appearance is a specially used domain to obtain positive social attention and a central self-evaluative dimension for women (Ferreira, Pinto-Gouveia, \& Duarte, 2011; Gilbert, 2002; Gilbert, Price, \& Allan, 1995; Myers \& Crowther, 2009; Troop, Allan, Treasure \& Katzman, 2003). In modern western culture, a thin body shape became a representation of female attractiveness and synonymous of physical and psychological positive attributes, such as beauty, health, success, power and happiness 
(Feingold, 1992; Kanazawa \& Kovar, 2004; Strahan, Wilson, Cressman, \& Buote, 2006; Webster \& Driskell, 1983; Wiseman, Gray, Mosimann, \& Ahrens, 1992). In this sense, the control over eating, weight and body image may operate as a strategy to enhance one's social status, compete for social advantages, such as being chosen for important social roles (e.g., friend, lover, team member), and to be valued by the social group (Gatward, 2007; Burkle, Ryckman, Gold, Thornton, \& Audesse, 1999). Actually, research has shown that body image dissatisfaction and the intense need to reach a thin body shape and fears of weight gaining, emerge in the context of a social rank mentality, characterized by feelings of inferiority, devaluation and ostracism derived from social comparisons based on physical appearance and shame (Ferreira et al., 2011; Pinto-Gouveia et al., 2012).

Shame is a powerful self-conscious and socially-focused emotion related to the dynamics of competition for social attractiveness. This means that shame arises in social interactions from perceptions of existing negatively in the mind of the others as an unattractive and undesirable social agent and is often accompanied by the internalization of this negative self-evaluative image of oneself (Gilbert, 2002, 2007; Tangney \& Dearing, 2002). A consistent body of research has associated shame with eating psychopathology both in nonclinical (e.g., Gee \& Troop, 2003; Murray, Waller, \& Legg, 2000; Sanftner, Barlow, Marschall, \& Tangney, 1995) and clinical samples (Cooper, Todd, \& Wells, 1998; Gee \& Troop, 2003; Grabhorn, Stenner, Stangier, \& Kaufhold, 2006; Skarderud, 2007; Swan \& Andrews, 2003).

Negative perceptions of one's social rank and the experience of the self as unable to generate positive images and affects in the minds of the others and as being vulnerable to their attack, exclusion or rejection, may arise from early shame experiences (Gilbert, 2007; Gilbert, Allan \& Goss, 1996; Tangney \& Dearing, 2002). 
Shame experiences (such as being criticized by a parent, bullied by a peer, putdown by a teacher or rejected by a lover) compose a primary threat to one's social self and selfidentity and can occur within the family, with attachment figures, or in the wider social domain, involving peers, teachers, and other social agents (Gilbert, 2007; Matos, PintoGouveia, \& Costa, 2011). These early shame events can be encoded as traumatic memories, eliciting symptoms of intrusion, hyperarousal and avoidance (Matos \& Pinto-Gouveia, 2010), and can become central to self-identity and life story (PintoGouveia, \& Matos, 2011). Research has also indicated that such traumatic and central shame memories have a significant impact on several psychopathological indicators, namely depression, anxiety and paranoia symptoms (Matos \& Pinto-Gouveia, 2010; Pinto-Gouveia \& Matos, 2011; Matos, Pinto-Gouveia, \& Duarte, 2012, in press; Matos, Pinto-Gouveia, \& Gilbert, 2012). Even though clinical accounts and some empirical data shed light on the role of early adverse experiences, such as weight-related teasing and bullying, in the proneness to shame and eating disorders' symptoms (Keith, Gillanders, \& Simpson, 2009; Van der Berg, Wertheim, Thompson, \& Paxton, 2002), the impact and phenomenology of early shame experiences on eating psychopathology remains to be investigated.

This study aimed at understanding the phenomenology of shame experiences in a sample of eating disorders patients. Furthermore, this study explores the traumatic features and the centrality to identity of early shame experiences with other social agents and with attachment figures. In addition, the present research investigates the associations between the traumatic and centrality qualities of shame memories with attachment figures and with other social agents, social rank perceptions defined by one's physical appearance, and key features of eating psychopathology and their severity. Prior research has highlighted eating psychopathology symptoms as means of 
competing in the social world and avoiding shame and a sense of inferiority derived from one's physical appearance (Ferreira et al., 2011; Pinto-Gouveia et al., 2012). Therefore, it is expected that traumatic and central shame memories involving others from the wider social domain (e.g., peers) would be more strongly linked to the overevaluation of eating, shape and weight and related pathological behaviours, in comparison to those involving attachment figures. In addition, the current study tests a mediational model in which it is predicted that the relationship between the traumatic and centrality features of early shame memories with other social agents and eating psychopathology severity would be mediated by perceptions of lower social rank based on one's physical appearance.

\section{Materials and methods}

\section{Participants}

Thirty-six patients with an eating disorder diagnosis participated in this study. Their age ranged from 14 to 49 years old and they presented a mean age of $25.19(S D=$ $8.44)$ and a mean of $12.83(S D=3.55)$ years of education. Three clinical groups were formed according to participants' clinical condition: $30.6 \%(n=11)$ patients presented Anorexia Nervosa, 41.7\% $(n=15)$ Bulimia Nervosa, and 27.8\% $(n=10)$ Eating Disorder Not Otherwise Specified. The patients' BMI ranged from 15.35 to 47.33, and the mean is $22.36(S D=8.14)$.

\section{Procedures}

The study sample is part of a wider research investigating the contribution of a social rank mentality and shame in the aetiology of eating disorders. 
The clinical sample was collected in outpatient mental health services, at an eating disorders Care Unit, after previous review and approval by the local Ethics Committee Boards.

First, participants were informed of the purpose and procedures of the research, that their participation was voluntary and that the data were confidential, and gave their informed consent. Clinical diagnoses were established using the structured clinical interview Eating Disorder Examination 16.0D (EDE 16.0D; Fairburn et al., 2008) which was administered by the second and third authors (who are clinical psychologists and trained researchers). Those patients who met the criteria for an eating disorder diagnosis (DSM IV; APA, 2000) were asked to answer a set of self-report questionnaires, including the Social Comparison through Physical Appearance Scale (Ferreira et al., 2013) used in the present study.

In a second session, the Shame Experiences Interview (SEI; Matos \& PintoGouveia, 2006) was administered in a clinical environment by the first author, who is a trained researcher and also clinical psychologist, with experience in eating disorders, and lasted on average 90 to 120 minutes. This second session took place approximately 1 to 2 weeks after the first one, depending on the patient's availability. With some patients presenting more severe symptomatology, an additional session was necessary to complete the SEI, in order to prevent bias due to fatigue or emotional activation. As part of the SEI, participants completed the self-report questionnaires measuring the shame memories' traumatic and centrality features.

Patients were excluded from the study if they did not demonstrate intellectual capacity to understand and respond appropriately to the test materials; and/or were experiencing current psychotic illness or presented an active (untreated) substance use disorder. 


\section{Measures}

Eating Disorder Examination 16.0D (EDE 16.0D; Fairburn, Cooper, \& O'Connor, 2008; Psychometric properties of the Portuguese Population by Ferreira, Pinto-Gouveia, \& Duarte, 2010) is a standardized interview that can be used for diagnosing eating disorders based on the DSM-IV-TR (2000) criteria, and allows the assessment of the frequency and intensity of core behavioural and psychological characteristics of eating disorders. It comprises four subscales, dietary restraint (e.g., restraint over eating, avoidance of eating, food avoidance, dietary rules), eating concern (e.g., preoccupations with food, eating or calories, fear of losing control over eating, guilt about eating), shape concern (e.g., preoccupation with shape or weight, fear of gaining weight, dissatisfaction regarding one's shape, discomfort about seeing or exposing one's body, feelings of fatness), and weight concern (e.g., importance of weight, concerns about shape or weight, desire to lose weight). It is considered a precise evaluation method with high values of internal consistency, of test-retest reliability, and of discriminative and concurrent validity (see Fairburn (2008) for a review).

Social Comparison through Physical Appearance Scale (SCPAS; Ferreira et al., 2013). The SCPAS is a brief and valid measure that allows the assessment of how individuals perceive themselves in a social context, having as a reference their physical appearance and assesses the subjective perception of women's attractiveness, social ranking and group fit. The participants are instructed to select a number, using a Likert scale ranging from 1 to 10 , which best translates the way they feel in relation to other people [e.g., 'When I physically compare myself with friends, colleagues and other known girls (proximal targets - Part A: Peers) /models, actresses or celebrities (distal 
targets - Part B: Models) I feel . . . Inferior/Superior, Left out/Accepted, Devalued/Valued']. Higher scores represent more favourable comparisons. The SCPAS presents a high internal reliability (.94 in Part A: Peers, and .96 in Part B: Models; Ferreira et al., 2011). Given the aims of the current study, the Part A: Peers was selected to integrate the analyses.

Shame Experiences Interview (SEI, Matos \& Pinto-Gouveia, 2006). The SEI is a semi-structured qualitative and quantitative interview designed to assess the phenomenology of a shame experience from childhood or adolescence. It measures emotional, cognitive, behavioural, motivational and contextual components of shame and its autobiographical/traumatic memory characteristics. The interview begins with an introduction explaining the concept of shame and giving examples of shame experiences from childhood and adolescence. It is divided into three main parts: In the first part, a significant shame memory that involved peers, teachers, strangers, or other people, is elicited and assessed regarding its phenomenological and memory characteristics. In the second part, participants are asked to recall a significant shame memory involving an attachment figure (father, mother or other caregiver), and its phenomenological and memory characteristics are evaluated. The third part measures the accessibility to positive and negative memories with attachment figures (i.e., number of positive/negative memories one recalls in a period of time). After each part, participants are asked to fill in a set of self-report questionnaires considering the shame memory elicited, measuring shame traumatic memory characteristics and centrality of shame memory. For the purpose of this study, we will only consider the scores from the self-report measures described below applied to the shame memory with peers, teachers, strangers or other people and to the shame memory with attachment figures. 
Impact of Event Scale - Revised (IES-R) was developed by Weiss \& Marmar (1997). The IES-R is a self-report instrument designed to measure current subjective distress for any specific life event, and distinctively in our study, in relation to the shame memory involving peers, teachers, strangers or others (IES-R_Others) and to the shame memory with attachment figures (IES-R_Attachment Figures). This scale has 22 items - 7 items having been added to the original 15-item IES (Weiss \& Marmar, 1997) rated on a 5-point Likert scale (0-4). The IES-R is composed of three subscales that measure the three main characteristics of traumatic memories: avoidance (e.g., "I stayed away from reminders of it"), intrusion (e.g., "Any reminder brought back feelings about $i t ")$ and hyperarousal (e.g., "I was jumpy and easily startled") that parallel the DSM-IV criteria for PTSD. In the original study, Cronbach alphas of the subscales ranged from .87 to .92 for intrusion, .84 to .86 for avoidance and .79 to .90 for hyperarousal (Weiss \& Marmar, 1997). The Portuguese version revealed a one-dimensional structure with sound psychometric properties (IES-R Total Cronbach's $\alpha=.96$; Matos, Pinto-Gouveia, \& Martins, 2011).

Centrality of Event Scale (CES; Berntsen \& Rubin, 2006; Portuguese version by Matos, Pinto-Gouveia, \& Gomes, 2010) assesses the extent to which a memory of a stressful event becomes a reference point for personal identity and for attribution of meaning to other experiences in a person's life. This self-report questionnaire consists of 20 items, rated on 5-point Likert scale (1-5), that measure the three interdependent characteristics of highly negative emotional memories: reference points for everyday inferences ("This event has coloured the way I think and feel about other experiences."), turning points in life stories ("I feel that this event has become a central part of my life story.") and components of personal identity ("I feel that this event has become part of my identity."). In this study, participants completed the CES in relation to the shame 
memory involving peers, teachers, strangers or others (CES_Others) and to the shame memory with attachment figures (CES_Attachment Figures). In its original study, the CES reported a high internal consistency (Cronbach's $\alpha=.94)$. A one-dimensional structure with good psychometric properties was found in the Portuguese version (CES_Total Cronbach's $\alpha=.96$; Matos et al., 2010).

Cronbach' alphas for all study variables are reported in Table 2.

\section{Data analysis}

Data analyses were performed using PASW (PASW; Predictive Analytics Software, version 18, SPSS Inc., Chicago, IL, USA).

In order to explore differences between the three groups in the variables studied, a series of one-way ANOVAs were conducted. Descriptive statistics (frequencies, means and standard deviations) were used to explore the phenomenology of the shame experiences recalled with others and with attachment figures (Howell, 2006). Paired Samples $t$ tests were conducted to test the significance of the differences between the traumatic and centrality features of shame memories involving others and involving attachment figures, considering different shame experiences for the same person (Howell, 2006).

Pearson correlation coefficients were performed to explore the association between shame memory variables, social comparison based on physical appearance and the severity of eating disorders symptomatology (Cohen, Cohen, West \& Aiken, 2003).

Two mediator analyses were conducted using linear regression models to test the effect of the mediator on the relationship between the predictor and the dependent variable. In each mediator analysis, social comparison based on physical appearance (with peers) was assumed to be the mediator on the relationship between the traumatic 
features (Model A) and the centrality of identity (Model B) of shame memories with others (predictor variables) and eating psychopathology severity (dependent variable; Baron and Kenny, 1986). Furthermore, the significance of the indirect effects was tested using a bootstrapping method developed by Preacher and Hayes (2008), which produces a confidence interval for the size of the indirect path. A statistically significant mediation effect exists if the values between the upper and lower confidence bound do not include zero.

\section{Results}

\section{Between groups comparisons}

In order to explore differences between the three clinical conditions groups in regard to the study variables, a multivariate general linear model was conducted (Table 1). Results indicated that the three groups did not differ regarding eating psychopathology indicators, shame memory and social ranking variables. Therefore, we considered only one group in subsequent analyses.

Means and standard deviations for the total eating disorders sample $(N=36)$ are presented in Table 2 .

\section{Description of shame memories phenomenology}

The phenomenology of the shame memories from childhood and adolescence with other social agents and with attachment figures was assessed regarding type of shame situation and 'shamer'. In regard to shame memory with others, the most frequent shame experiences reported were those where participants were criticized, teased or rejected $(n=16,44.4 \%)$ and events where an aspect related to their weight, body or physical appearance was negatively commented on or criticized by others $(n=$ 
$9,25 \%$ ), followed by situations where they felt shame for having a negative personal attribute or characteristics or devaluing behaviour exposed in front of others $(n=5$, 13.9\%). Less frequent shame experiences were: feeling shame due to being negatively compared with significant others $(n=2,5.6 \%)$ and shame of one's family status $(n=2$, 5.6\%). Finally, two participants $(5.6 \%)$ recalled situations where they were physically (e.g., victims of bullying) or sexual abused.

When asked about who shamed them in the experience described, the majority of the participants $(n=15,41.6 \%)$ remembered events where they were shamed by their peers (i.e., friends and colleagues), $27.8 \%(n=10)$ identified themselves as the shamers (i.e., for being responsible of having a negative and devaluing personal attribute, characteristic or behaviour exposed in public contexts in front of others, i.e., non-attachment figures, such as peers), $22.2 \%(n=8)$ by other people (e.g., teacher, relatives) and $8.3 \%(n=3)$ several of the aforementioned shamers (e.g., peers and teacher, peers and friends). Regarding shamer(s)' characteristics, the shamer(s) was typically defined as being someone they liked and/or knew, with the same age, equal in social rank and gender (i.e., female).

In regard to shame memories with attachment figures, most participants described situations where they were criticized, put-down or felt rejected by a caregiver $(n=11,31.4 \%)$. The second most frequent shame experiences recalled were of psychological or emotional abuse (e.g., neglect, verbal abuse; $n=7,20 \%$ ). Participants also recalled events where the attachment figure criticized or made comments about their weight, body or physical appearance $(n=5,14.3 \%)$, reflected shame situations (i.e., feeling shame due to characteristics or behaviour of the attachment figure, or due to family social status; $n=7,20 \%$ ), events where they had negative personal attributes or characteristics or devaluing behaviour exposed in front of the attachment figure(s) ( $n$ 
$=3,8.6 \%$ ). Two participants recalled situations where they were physically abused by the attachment figure.

Concerning the attachment figure who shamed them in that shame event, $42.9 \%$ $(n=15)$ participants identified the father and $40 \%(n=14)$ the mother as the shamers, $8.6 \%(n=3)$ were shamed by both parents and $8.6 \%(n=3)$ by another caregiver. Given that these shamers were attachment figures, and thus assumed to be loved ones, older and dominant, intimacy, age and power features were not directly assessed.

In addition, descriptive statistics showed that these recalled shame experiences, with other social agents and with attachment figures, revealed traumatic memory characteristics (i.e., symptoms of intrusion, hyperarousal and avoidance) and were regarded as central to one's identity and life story. Paired-samples $t$ tests revealed no significant differences between shame memories with others and with attachment figures regarding traumatic features $[t(34)=0.99, p=.332]$ and centrality to personal identity $[t(34)=1.03, p=.313]$.

\section{Correlations}

Pearson's correlation coefficients (two-tailed) are presented in Table 2. Results showed that shame traumatic memory with others was significantly, and moderately, correlated with dietary restraint, eating and shape concerns, and with overall levels of eating psychopathology. With stronger magnitude correlations, centrality of shame memory with others was also significantly correlated with dietary restraint and concerns regarding eating, weight and shape, and with the global indicator of eating psychopathology.

Regarding shame memories with attachment figures, no significant correlations were found between shame memory traumatic and centrality features and eating 
psychopathology indicators, with the only exception of the weak correlation between shame traumatic memory and the dietary restraint subscale.

Furthermore, the traumatic and centrality features of shame memory with others were strongly and inversely associated with social comparison based on physical appearance with peers. On the other hand, traumatic and central shame memories with attachment figures were not significantly correlated with this variable.

Finally, social comparison in relation to peers based on the physical appearance domain was inversely, and moderately to strongly, correlated with all the EDE subscales and global score.

In addition, age of the situation was controlled for in partial correlations. The results maintained the same pattern and therefore this variable was not further considered in the analysis.

\section{The mediator effect of social comparison on the relationship between shame memories with others and eating psychopathology severity}

In order to further explore these findings, two mediator analyses were conducted using linear regression models to test whether social rank perceptions based on one's physical appearance would mediate the relationship between 1) traumatic shame memories with others and 2) the centrality of shame memories, and eating psychopathology severity (Figure 1).

Preliminary data analyses indicated that these data were suitable for regression analyses following the assumptions of normality, linearity, homoscedasticity, independence of errors and multicollinearity. In addition, the correlation coefficients between these variables point to adequate effect sizes [ranging from $.21(r=.46)$ to .45 
$(r=.67)]$. Furthermore, our sample had the required size to conduct the analysis to achieve a large effect size when considering two predictors (Miles \& Shevlin, 2001).

\section{Social comparison as a mediator between shame traumatic memories and eating psychopathology severity}

A regression analysis was conducted with shame traumatic memory with others entered as the independent variable and eating psychopathology severity as the dependent variable. The model was significant $\left[F_{(1,31)}=8.83, p=.006\right]$, accounting for $22.2 \%$ of eating psychopathology severity $\left(\beta_{\mathrm{IES}-\mathrm{R}}=.47, p=.006\right)$. The next analysis was conducted to examine whether shame traumatic memory with others predicted social comparison through physical appearance with peers. The model was also significant $\left[R^{2}\right.$ $\left.=.33, F_{(1,30)}=15.23, p<.001\right]$ with $\beta_{\text {IES-R }}=-.57(p<.001)$. Finally, a regression analysis was performed to determine whether the proposed mediator significantly predicted eating psychopathology severity. We entered shame traumatic memory with others and social comparison through physical appearance with peers as the independent variables and eating psychopathology severity as the dependent variable. The final model was significant $\left[F_{(1,30)}=10.27, p=.003\right]$, accounting for $42 \%$ of the variance in eating psychopathology severity. Results indicate that when the mediator is added in, the predictor $\beta$ is reduced to $.16(p=.358)$ and is no longer significant, while the mediator emerges as the only significant predictor $\left(\beta_{\mathrm{SCPAS}}=-.54, p=.003\right.$; see Figure 1). Bootstrapping method indicated that the indirect path through social comparison defined by one's physical appearance was statistically significant since the $95 \%$ Confidence Interval (bias corrected) did not include zero (.011 to .297). So, social rank perceptions defined by one's physical appearance in comparison to peers fully mediates the effect of shame traumatic memory with others on eating psychopathology severity. 


\section{Social comparison as a mediator between centrality of shame memories and eating psychopathology severity}

Regarding the centrality of shame memories with others, the same procedure was conducted. First, when centrality of shame memory with others was entered as the independent variable and eating psychopathology severity as the dependent variable, a significant model was produced $\left[F_{(1,31)}=18.84, p<.001\right]$, accounting for $37.8 \%$ of the independent variable variance $\left(\beta_{\mathrm{CES}}=.62 ; p<.001\right)$. On the second step, a regression analysis was conducted to examine whether centrality of shame memory with others predicted unfavourable social comparisons based on physical appearance. The model was also significant $\left[R^{2}=.45, F_{(1,31)}=25.24, p<.001\right]$ with $\beta_{\mathrm{CES}}=-.67(p<.001)$. Finally, a regression analysis was performed to determine whether the proposed mediator significantly predicted eating psychopathology severity. Centrality of shame memory with others and social comparisons based on physical appearance were entered as the independent variables and eating psychopathology severity as the dependent variable. The final model was significant $\left[F_{(1,30)}=5.10, p=.031\right]$, accounting for $46.8 \%$ of the independent variable. Results indicate that when the mediator is added in, the predictor $\beta$ is reduced and is no longer significant $\left(\beta_{\mathrm{CES}}=.34, p=.065\right)$ and the mediator emerges as the only significant predictor of eating psychopathology severity $\left(\beta_{\mathrm{SCPAS}}=-.41, p=.031\right.$; see Figure 1$)$. The significance of this indirect path was confirmed through the Bootstrapping method, which showed that the 95\% Confidence Interval (bias corrected) did not include zero (.001 to .040). This suggests that social comparison with peers based on the physical appearance domain also fully mediates the effect of centrality of shame memory with others on eating psychopathology severity. 


\section{Discussion}

Shame is a common experience among patients with eating disorders, who often report feelings of inferiority, unworthiness, defect, inadequacy when comparing themselves with others (e.g., Gee \& Troop, 2003; Grabhorn et al., 2006; Skarderud, 2007). Research has consistently shown that negative self-perceptions may emerge from early shame experiences, in the wider social domain or within the family, where the self was felt as existing negatively for others (Gilbert, 2002, 2007; Matos, et al., 2012). These adverse and powerful emotional experiences can become central to one's selfidentity and life story and function as traumatic memories (Matos \& Pinto-Gouveia, 2010; Pinto-Gouveia \& Matos, 2011). Nevertheless, the role of early shame experiences and their characteristics in patients with eating disorders remained to be investigated. Thus, the current study aimed to explore the phenomenology of shame memories and their qualities of traumatic and centrality to identity in relation to eating psychopathology severity.

Results showed that patients with anorexia nervosa, bulimia nervosa and EDNOS did not significantly differ regarding EDE subscales and global scores, shame memory traumatic and centrality qualities, and social comparison based on physical appearance. Furthermore, and as expected, the three clinical groups presented EDE subscales and global scores similar to those reported in other studies using eating disorders samples (Pinto-Gouveia et al., 2012) and above the recommended cut points for clinical diagnosis in the Portuguese population (Ferreira, 2012). Similarly to previous studies, patients with eating disorders compared themselves more unfavorably

with others than women from the general population (Ferreira et al., 2011). Regarding shame memory features, in the present study sample, patient' scores in shame memories' traumatic and centrality features, with others and with attachment figures, 
were higher than those found in recent studies using non-clinical samples (Matos \& Pinto-Gouveia, 2010; Matos, Pinto-Gouveia, \& Costa, 2011; Pinto-Gouveia \& Matos, 2011). These results indicate that patients with eating disorders recall their early shame experiences, involving others and attachment figures, as especially traumatic and central memories to their self-identity, as well as regard themselves as being significantly worse when socially comparing themselves with peers based on the physical appearance domain.

Our findings revealed that the most frequent shame experiences recalled by patients with eating disorders were situations where they were criticized, put-down or felt rejected by a caregiver or by other social agents, mostly peers. Specific shame experiences where the physical appearance was the focus of teasing or criticism were also remembered as the most significant shame events occurring in their childhood or adolescence. These results add to recent research in shame memories' phenomenology which showed that experiences of criticism, rejection and slur, and also those involving negative comments and criticism targeting one's body image were prevalent and significant shame experiences reported in clinical and non-clinical samples (Matos, 2012; Matos, Pinto-Gouveia, \& Duarte, 2012).

Furthermore, the results showed that eating psychopathology key features as well as social comparison based on the physical appearance domain were significantly correlated with the traumatic and centrality qualities of shame memories with others, with the former presenting the stronger associations. On the other hand, the traumatic and centrality qualities of shame memories with attachment figures revealed nonsignificant or weak correlations with eating psychopathology and social comparison based on physical appearance. This is an interesting finding as it highlights that in patients with eating disorders, more than early shame experiences with attachment 
figures, it is those involving others from the wider social domain (i.e., peers), that become traumatic and central memories to self-identity, that are linked to eating psychopathology severity and with their sense of inferiority in relation to others. This is consistent with and adds to the existing research regarding the role of early negative experiences, in the form of abuse, bullying and weight-related bullying and teasing, in the vulnerability to eating disorders (Keith et al., 2009, Van der Berg et al., 2002; Menzel et al., 2010). In addition, these results complement prior research on how shame memories can become traumatic and central to one's identity and life story and their impact on wide range on psychopathology symptoms (Matos \& Pinto-Gouveia, 2010; Matos et al., 2011; Matos, Pinto-Gouveia, \& Duarte, 2012, in press; Pinto-Gouveia \& Matos, 2011), offering new insights on how these memories operate in eating disorders.

Finally, mediational tests' results indicated that social rank perceptions based on physical appearance fully mediated the relationship between shame memories with traumatic and centrality features and the severity of eating psychopathology. This shows that the impact of traumatic and central shame memories on eating psychopathology is not direct but fully operates through their effect on diminishing one's perception of social rank when comparing oneself with peers (e.g., friends, colleagues and other known girls) based on the physical appearance domain. These findings imply that the extent to which these shame memories with peers impact on current eating psychopathology severity depends on how these traumatic and central to identity memories become internalized into a sense of self as worthless, inferior, inadequate and different when physical appearance is used as a means to define one's social rank perceptions. Such results extend prior research highlighting how the need to control one's body image emerges as a means to avoid shame and as an attempt to belong, be 
accepted and valued by the social world (Ferreira et al., 2011; Pinto-Gouveia et al., 2012).

This research offers important insights for clinical work with eating disorders patients. Our findings suggest that therapists should carefully assess these patients early shame experiences, especially those occurring with peers, and how these became structured as traumatic and central memories to self-identity. Also, specific work targeting these shame memories that focuses on ameliorating their traumatic nature and reconstructing their autobiographical meaning to self-identity might help to reduce their impact on one's sense of inferiority derived from social comparisons based on physical appearance that, in turn, fuel the core features of eating disorders.

These novel findings cannot be considered without taking into account some limitations. First, the generalization of these results is constrained by the small sample size of our study. This limitation is related to the nature of the study itself, which required an extensive evaluation through two clinical semi-structured interviews, that are time consuming and require specific training and clinical experience with eating disorders. At the same time, the use of these interviews constitutes a major strength of the study providing the collection of novel, rich and detailed phenomenological data on shame experiences in eating disorders patients. In addition, the statistical approach to the data took this sample size limitation into account and the results achieved had adequate power (e.g., Miles \& Shevlin, 2001).

Secondly, the retrospective nature of our data limits causal conclusion to be drawn from findings. In the future, these findings should be confirmed in studies using longitudinal designs. Nevertheless, a major strength of this innovative research lies on the use of structured clinical interviews which warranted a rigorous assessment methodology. 
This is the first study to explore the phenomenology of early shame experiences in eating disorders patients, and to emphasize the role of traumatic and central shame memories occurring with agents from the wider social domain (e.g., peers), in core maintenance mechanisms and features of eating disorders. 


\section{References}

American Psychiatric Association (2000). Diagnostic and statistical manual of mental disorders (4th ed. - TR). Washington, DC: Task Force.

Baron, R. M. \& Kenny, D. A. (1986). The moderator-mediator variable distinction in social psychological research: Conceptual, strategic, and statistical considerations. Journal of Personality and Social Psychology, 51, 1173-1182. doi: 10.1037/0022-3514.51.6.1173

Berntsen, D., \& Rubin, D. C. (2006). Centrality of event scale: A measure of integrating a trauma into one's identity and its relation to post-traumatic stress disorder symptoms. Behaviour Research and Therapy, 44, 219-231. doi: 10.1016/j.brat.2005.01.009

Burkle, M. A., Ryckman, R. M., Gold, J. A., Thornton, B., \& Audesse, R. J. (1999). Forms of competitive attitude and achievement orientation in relation to disordered eating. Sex Roles, 40(11-12), 853-870. doi: 10.1023/A:1018873005147

Cohen, J., Cohen, P. West, S. \& Aiken, L. (2003). Applied multiple regression/correlation analysis for the behavioural sciences (3th ed.). New Jersey: Lawrence Erlbaum Associates.

Cooper, J. M., Todd, G., \& Wells, A. (1998). Content, origins, and consequences of dysfunctional beliefs in anorexia nervosa and bulimia nervosa. Journal of Cognitive Psychotherapy, 12, 213-230. Retrieved from http://www.springerpub.com/product/08898391

Fairburn, C. G. (2008). Cognitive behavior therapy and eating disorders. New York: Guilford Press. 
Fairburn, C. G., Cooper Z., \& O’Connor, M. E. (2008). Eating Disorder Examination (Edition 16.0D). In C. G. Fairburn (Ed.), Cognitive Behavior Therapy and Eating Disorders (pp. 265-308). New York: The Guilford Press.

Fairburn, C. G., \& Harrison, P. J. (2003). Eating disorders. The Lancet, 361(9355), 407416. doi: 10.1016/S0140-6736(03)12378-1

Feingold, A. (1992). Good-looking people are not what we think. Psychological Bulletin, 111(2), 304-341. doi:10.1037/0033-2909.111.2.304.

Ferreira, C. (2012). O peso da magreza: O corpo como um instrumento de ranking social [The weight of thinness: The body as a social rank instrument] (Unpublished Doctoral Dissertation). University of Coimbra, Coimbra, Portugal.

Ferreira, C., Pinto-Gouveia, J., \& Duarte, C. (2010). Dados psicométricos da versão portuguesa da Eating Disorder Examination 16.0D [Psychometric data of the Portuguese version of the Eating Disorder Examination 16.0D]. Manuscript in preparation.

Ferreira, C., Pinto-Gouveia, J., \& Duarte, C. (2013). Physical appearance as a measure of social ranking: The role of a new scale to understand the relationship between weight and dieting. Clinical Psychology and Psychotherapy, 20, 55-66. doi: 10.1002/cpp.769.

Gatward, N. (2007). Anorexia nervosa: An evolutionary puzzle. European Eating Disorders Review, 15, 1-12. doi: 10.1002/erv.718.

Gee, A., \& Troop, N. N. (2003). Shame, depressive symptoms and eating weight and shape concerns in a nonclinical sample. Eating and Weight Disorders, 8, 72-75. Retrieved from http://www.kurtis.it/ewd/en/ 
Gilbert, P. (2002). Body shame: A biopsychosocial conceptualisation and overview, with treatment implications. In P. Gilbert \& J. Miles (Eds.), Body shame: Conceptualisation, research and treatment (pp. 3-54). London: Brunner.

Gilbert, P. (2007). The evolution of shame as a marker for relationship security. In J. L. Tracy, R. W. Robins \& J. P. Tangney (Eds.), The Self-conscious emotions: Theory and research (pp. 283-309). New York: Guilford Press.

Gilbert, P., Allan, S., \& Goss, K. (1996). Parental representations, shame, interpersonal problems, and vulnerability to psychopathology. Clinical Psychology and Psychotherapy, 3, 23-34. doi: 10.1002/(SICI)1099-0879(199603)3:1<23::AIDСРP66>3.0.CO;2-O

Gilbert, P., Price, J. S., \& Allan, S. (1995). Social comparison, social attractiveness and evolution: How might they be related? New Ideas in Psychology, 13, 149-165. doi: 10.1016/0732-118X(95)00002-X

Goss, K. P., \& Gilbert, P. (2002). Eating disorders, shame and pride: a cognitivebehavioural functional analysis. In P. Gilbert \& J. Miles (Eds.), Body shame: Conceptualization, research \& treatment (pp. 219-255). Hove, UK: BrunnerRoutledge.

Grabhorn, R., Stenner, H., Stangier, U. \& Kaufhold, J. (2006). Social anxiety in anorexia and bulimia nervosa: The mediating role of shame. Clinical Psychology and Psychotherapy, 13, 12-19. doi: 10.1002/cpp.463

Herzog, W., Deter, H. C., Fiehn, W., \& Petzold, E. (1997). Medical findings and predictors of long-term physical outcome in anorexia nervosa: A prospective 12year follow-up study. Psychological Medicine, 27(2), 269-279. doi: $10.1017 / \mathrm{S} 0033291796004394$ 
Howell, D. (2006). Statistical methods for psychology (6th ed.). USA: Thomson Wadsworth.

Kanazawa, S., \&. Kovar, L. J. (2004). Why beautiful people are more intelligent. Intelligence, 32, 227-243. doi: 10.1016/j.intell.2004.03.003.

Keith, L., Gillanders, D., \& Simpson, S. (2009). An exploration of the main sources of shame in an eating-disordered population. Clinical Psychology and Psychotherapy, 16, 317-327. doi: 10.1002/cpp.

Matos, M. (2012). Shame memories that shape who we are (Unpublished Doctoral Dissertation). University of Coimbra, Coimbra, Portugal.

Matos, M. \& Pinto-Gouveia, J. (2006). The shame experiences interview. Unpublished manuscript.

Matos, M. \& Pinto-Gouveia, J. (2010). Shame as a traumatic memory. Clinical Psychology and Psychotherapy, 17(4), 299-312. doi: 10.1002/cpp.659.

Matos, M., Pinto-Gouveia, J., \& Costa, V. (2011). Understanding the importance of attachment in shame traumatic memory relation to depression: The impact of emotion regulation processes. Clinical Psychology and Psychotherapy. Advance online publication. doi: 10.1002/cpp.786.

Matos, M., Pinto-Gouveia, J., \& Duarte, C. (2012). Above and beyond emotional valence: The unique contribution of the central and traumatic shame memories to psychopathology vulnerability. Memory, 20 (5), 461-477. doi: $10.1080 / 09658211.2012 .680962$.

Matos, M., Pinto-Gouveia, J., \& Duarte, C. (in press). Internalizing early memories of shame and lack of safeness and warmth: The mediating role of shame on depression. Behavioural and Cognitive Psychotherapy. (In press). 
Matos, M., Pinto-Gouveia, J., \& Gilbert, P. (2012). The effect of shame and shame memories on paranoid ideation and social anxiety. Clinical Psychology and Psychotherapy.Advance online publication. doi: 10.1002/cpp.1766.

Matos, M., Pinto-Gouveia, J., \& Gomes, P. (2010). A centralidade das experiências de vergonha: Estudo das propriedades psicométricas da versão portuguesa da Escala da Centralidade do Acontecimento [The centrality of shame experiences: Study of the psychometric properties of the Portuguese version of the Centrality of Event Scale]. Psicologia, XXIV (1), 73-95.

Matos, M., Pinto-Gouveia, J., \& Martins, S. (2011). O impacto traumático de experiências de vergonha: Estudo das propriedades psicométricas da versão portuguesa da Impact of Event Scale-Revised [The traumatic impact of shame experiences: Study of the psychometric properties of the Portuguese version of the Impact of Event Scale-Revised]. Psychologica, 54, 413-438.

Menzel, J., Schaefer, L., Burke, N., Mayhew, L., Brannick, M., \& Thompson, J. (2010). Appearance-related teasing, body dissatisfaction, and disordered eating: A meta-analysis. Body Image, 7(4), 261-270. doi: 10.1016/j.bodyim.2010.05.004.

Miles, J. \& Shevlin, M. (2001). Applying regression and correlation: a guide for students and researchers. London: Sage Publications.

Murray, C., Waller, G., \& Legg, C. (2000). Family dysfunction and bulimic psychopathology: The mediating role of shame. International Journal of Eating Disorders, 28, 84-89. doi: 10.1002/(SICI)1098-108X(200007)

Myers, T. A., \& Crowther, J. H. (2009). Social comparison as a predictor of body dissatisfaction. A meta-analytic review. Journal of Abnormal Psychology, 115(4), 683-698. doi:10.1037/a0016763. 
Pinto-Gouveia, J., Ferreira, C., \& Duarte, C. (2012). Thinness in the pursuit for social safeness: An integrative model of social rank mentality to explain eating psychopathology. Clinical Psychology and Psychotherapy. Advance online publication doi: $10.1002 /$ cpp. 1820

Sanftner, J. L., Barlow, D. H., Marschall, D. E., \& Tangney, J. P. (1995). The relation of shame and guilt to eating disorder symptomatology. Journal of Social and Clinical Psychology, 14, 315-324. doi: 10.1521/jscp.1995.14.4.315

Skarderud, F. (2007). Shame and pride in anorexia nervosa: A qualitative descriptive study. European Eating Disorders Review, 15, 81-97. doi: 10.1002/erv.774

Strahan, E. J., Wilson, A. E., Cressman, K. E., \& Buote, V. M. (2006). Comparing to perfection: How cultural norms for appearance affect social comparisons and self image. Body Image, 3, 211-227. doi: 10.1016/j.bodyim.2006.07.004.

Swan, S. \& Andrews, B. (2003). The relationship between shame, eating disorders and disclosure in treatment. The British Journal of Clinical Psychology, 42, 367383. doi: $10.1348 / 014466503322528919$

Tangney, J. P., \& Dearing, R. L. (2002). Shame and guilt. New York: Guilford Press.

Troop, N.A., Allan, S., Treasure, J.L., \& Katzman, M. (2003). Social comparison and submissive behaviour in eating disorders. Psychology and Psychotherapy: Theory, Research and Practice, 76, 237-249. doi: $10.1348 / 147608303322362479$

Van den Berg, P., Wertheim, E.H., Thompson, J.K., \& Paxton, S.J. (2002). Development of body image, eating disturbance, and general psychological functioning in adolescent females: A replication using covariance structure modeling in an Australian sample. International Journal of Eating Disorders, 32, 46-51. doi: 10.1002/eat.10030 
Webster J., \& Driskell, J. E. (1983). Beauty as status. American Journal of Sociology, 89(1), 140-165. doi: 10.1086/227836

Weiss, D. S., \& Marmar, C. R. (1997). The Impact of Event Scale - Revised. In J. P. Wilson, \& T. M. Keane (Eds.), Assessing Psychological trauma and PTSD (pp. 399-411). New York: Guilford Press.

Wiseman, C. V., Gray, J. J., Mosimann, J. E., \& Ahrens, A. H. (1992). Cultural expectations of thinness in women: An update. International Journal of Eating Disorders, 11, 85-89. doi:10.1002/1098-108X(199201). 
Table 1

Comparisons between the three clinical groups regarding the study variables

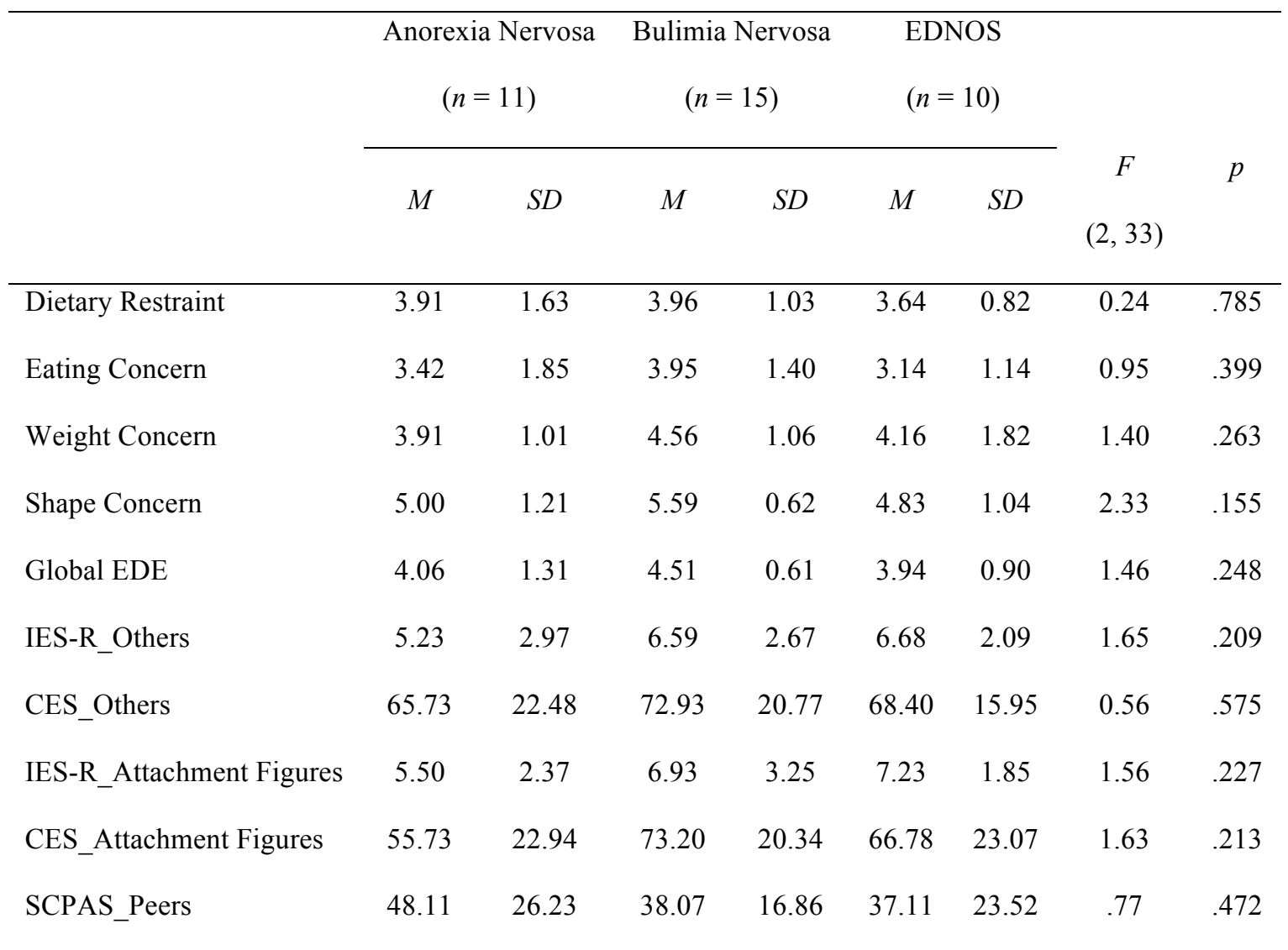

Key. Global EDE = Eating Disorder Examination global score; IES-R = Shame traumatic memory; CES

$=$ Centrality of shame memories; SCPAS_Peers = Social Comparison through Physical Appearance Scale, Peers subscale. 
Table 2

Means (M), Standard Deviations (SD), Cronbach alphas and Intercorrelation scores on self-report measures $(N=36)$

Measure

$\begin{array}{llllllll}M & S D & \alpha & 1 & 2 & 3 & 4 & 5\end{array}$

6

7

8

1. Dietary Restraint

$\begin{array}{llll}3.86 & 1.17 \quad .68 \quad-\end{array}$

2. Eating Concern

$\begin{array}{lllll}3.56 & 1.49 & .69 & .31 \quad-\end{array}$

3. Weight Concern

$\begin{array}{llllll}4.25 & 1.29 & .54 & .24 & .53 * * & -\end{array}$

4. Shape Concern

$\begin{array}{lllllll}5.20 & 0.99 & .78 & .55^{* *} & .60 * * * & .61 * * * & -\end{array}$

5. Global EDE

$\begin{array}{llllllll}4.22 & 0.96 & .84 & .65 * * * & .82 * * * & .78 * * * & .86 * * *\end{array}$

6. IES-R_Others

$\begin{array}{llllllll}6.20 & 2.63 & .93 & .37 * & .37 * & .28 & .43 * * & .46 * *\end{array}$

7. CES_Others

$69.47 \quad 19.80 \quad .96 \quad .47 * * \quad .51 * * \quad .40 * \quad .58 * * * \quad .63 * * * \quad .83 * * * \quad-$

8. IES-R_Attachment Figures

$\begin{array}{lllllllllll}6.55 & 2.71 & .94 & .33 * & .24 & .04 & .27 & .28 & .69 * * * & .59 * * * & -\end{array}$

9. CES_Attachment Figures $\begin{array}{llllll}66.06 & 22.53 & .97 & .22 & .24\end{array}$ $24 \quad-.04 \quad .20$ .21 $.37 * \quad .46^{* *} \quad .71 * * *$

10.SCPAS_Peers

$\begin{array}{llllllllllll}40.55 & 21.37 & .96 & -.50 * * & -43 * & -.45 * * & -.63 * * * & -.64 * * * & -.57 * * * & -.67 * * * & -.29 & -.26\end{array}$ Note. ${ }^{*} p<.050 .{ }^{* *} p<.010 . * * * p<.001$. Global EDE = Eating Disorder Examination global score; IES-R = Shame traumatic memory; CES $=$ Centrality of shame memories; SCPAS_Peers = Social Comparison through Physical Appearance Scale, Peers subscale. 


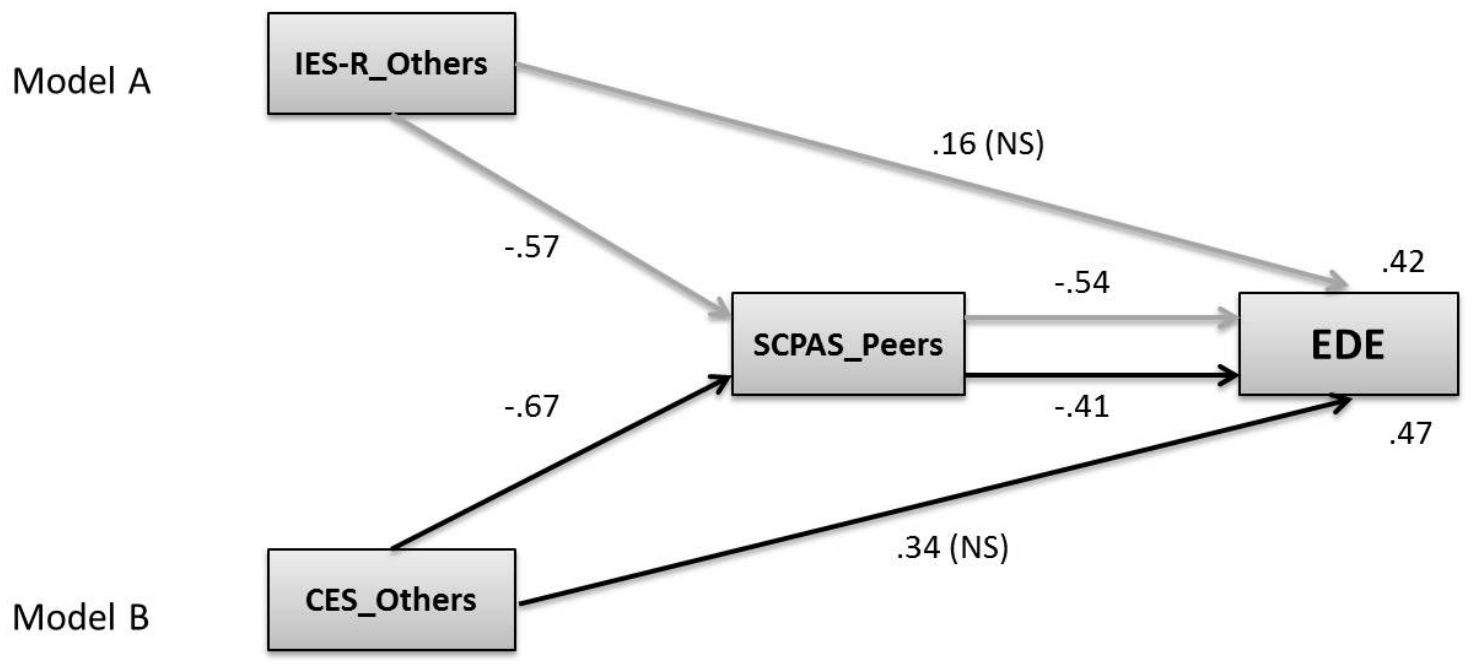

Figure 1. The mediational effect of social comparison based on physical appearance (SCPAS_Peers) on the relationships between the traumatic (IES-S_Others; Model A) and centrality (CES_Others; Model B) qualities of shame memories involving other social agents and eating disorders severity (EDE) 